\title{
KEY-POINT BASED COPY-MOVE FORGERY DETECTION AND THEIR HYBRID METHODS: A REVIEW
}

\author{
Harpreet Kaur ${ }^{1 *}$, Jyoti Saxena ${ }^{2}$ and Sukhjinder Singh ${ }^{3}$ \\ ${ }^{* 1}$ Research Scholar, ${ }^{2}$ Professor and ${ }^{3}$ Assistant Professor \\ *1,2,3 Deptt. of Electroics and Communication Engineering, GZS-PTU Campus, Bathinda, Punjab, India.
}

*Corresponding Author: -

\begin{abstract}
: -
Copy-move image forgery is one of the tampering techniques that need to be tackled with. Many copy-move forgery detection techniques such as exhaustive search, block and key-point matching based methods have been proposed for the detection of copy-move image forgery. Although key-point based methods were found better than block-based methods in terms of computational efficiency, space complexity and robustness against rotation and scaling. However, key-point based methods also possess a number of limitations. So, researchers have proposed many integrated methods to cope up with the limitations of key-point based methods and to make copy move forgery detection more reliable. In this paper, key-point based methods such as SIFT, SURF, ORB and their integrated methods are reviewed.
\end{abstract}

Keywords: - Block based, copy-move image forgery, key-point based, SIFT, SURF and ORB.

\section{(c) $(\$)$ (i)}




\section{INTRODUCTION}

The availability and easy access of powerful image editing software tools like adobe photoshop, corel paintshop, XnView and Proshow Gold etc. have given rise to large amount of doctored images which are indistinguishable from the authentic images. The manipulation of the digital image to hide some important or meaningful information or to create misleading images without leaving any visual traces is called image forgery. The manipulation of an image may include scaling, rotation, brightness adjustment, blurring, contrast enhancement or any combination of above [2]. Image forgery can be broadly classified in three categories namely image forgery using splicing, copy move image forgery and image resampling [3]. This paper focuses on copy-move image forgery and its detection methods. The paper is organized as follows. In section-2, copy-move image forgery and its detection techniques are discussed. Section-3 reviews the existing key-point based methods and their integrated methods. Section-4 presents discussion and conclusion.

\section{COPY-MOVE IMAGE FORGERY DETECTION}

Copy-move is an image forgery technique in which parts of an original image, after some possible geometric and illumination adjustments, are copied, moved to a desired location in the same image and pasted (e.g. refer figure 1.1). The main aim of copy-move image forgery is to hide certain details or to duplicate some aspects of an image [4].

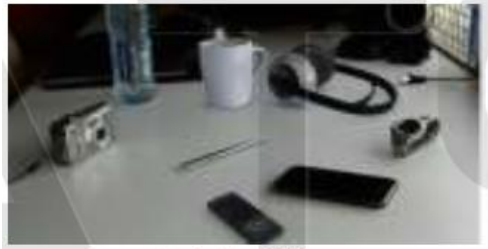

a) Original Image image

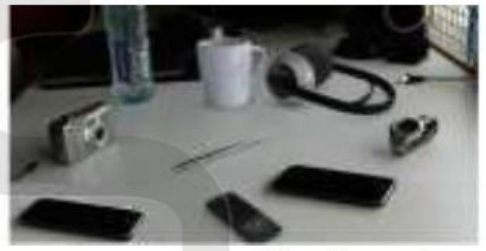

b) Forged

Figure1.1: An example of copy moves image forgery

As the copied part belongs to the image itself, its properties such as RGB values, texture properties etc. remain the same. This correlation makes detection of copy-move forgery difficult [5].

\subsection{Copy-Move Image Forgery Detection Methods}

Copy-move forgery detection techniques are broadly classified into two categories namely block based and key- point based methods as depicted in figure 2.1 .

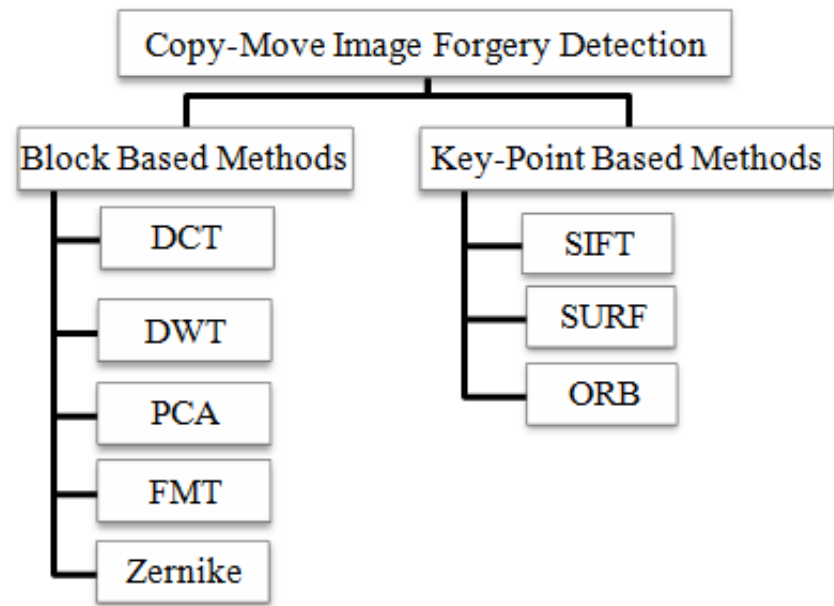

Figure2.1: Classification of copy move image forgery detection techniques

\subsubsection{Block based Methods}

The block-based methods subdivide the image into blocks such as rectangular, circular etc. for feature extraction. The block-based methods work as following steps [6]:

Step 1: Convert the image into gray scale image.

Step 2: Divide the image into overlapping blocks.

Step 3: Extract features from each block using block-based technique.

Step 4: Match similar features.

Step 5: Forgery decision is taken based upon some predefined threshold value.

The most commonly used block-based methods are DCT (Discrete Cosine Transform), PCA (Principal Component Analysis), DWT (Discrete Wavelet Transform), DyWT (Dyadic Wavelet Transform), FMT (Fourier Mellin Transform), Zernike etc. 


\subsubsection{Key-point based Methods}

Key-point based methods compute the feature vectors for regions with high entropy in an image. The working of keypoint based methods is given below [6].

Step 1: Convert image into grey scale image.

Step 2: Compute the features only on image regions with high entropy, without any image subdivision.

Step 3: Similar feature vectors within the image are matched

Step 4: Forgery is reported if regions of such matches cluster into larger areas.

Step 5: Some form of post processing may be done such as filtering, edge detection etc.

SIFT (Scale in-variant Feature Transform), SURF (Speed Up Robust Features), ORB (Oriented FAST (Features from Accelerated Segment Test) and Rotated BRIEF+++++ etc. are the examples of key-point based methods.

\subsubsection{Research Issues}

In this paper, main focus is on key-point based methods. Although key-point based methods have numerous advantages as compared to block-based methods such as high computational efficiency, robustness against geometric transformations like scaling, rotation, illumination variations etc. [8]. Yet there are many issues related to key-point based methods and these are liste+d below [7, 8 and 20].

> Key-point based methods such as SIFT; SURF alone is not able to detect flat copied regions i.e. region with highly uniform texture.

$>$ High False positive rate (FPR) of the detection results is also a major issue and need to be resolved.

$>$ Key-point based methods are unable to distinguish between original region and duplicate region.

$>$ Time taken by these methods to detect forgery can be reduced further by using appropriate methods.

$>$ These methods cannot handle mirror reflection transformation.

To handle with the above listed problems, a number of hybrid techniques have been proposed by researchers from time to time. A review of key-point based methods and hybrid techniques based on them is presented in the next section.

\section{RELATED WORK}

Various researchers have developed many methods to detect copy move image forgery. Out of those methods, key-point based methods (SIFT, SURF and ORB) and their integrated methods have been briefly discussed in the following subsections

\subsection{SIFT and its integrated Methods}

Huang et al., (2008) proposed an effective method based on Scale Invariant Feature Transform (SIFT) which was based on feature matching to detect copy-move forgery in digital images. To detect the original and the tampered region in the image SIFT descriptors were extracted and matched. Although the proposed method was found accurate and robust against different kinds of post processing operations such as JPEG compression, rotation, noise, scaling etc. and combination of all these operations. However, it still needs some improvements in detecting forgeries of small sized regions [9]. E. Ardizzone et al., (2010) proposed a cluster method to find multiple copies of the same region. They aimed to match clusters of keys- points (extracted by SIFT) instead of single points only. Cluster method resulted in better percentage of correct matching and also eliminated false matching [10]. A method based on SIFT was proposed by Amerini.I et al., (2011) which was capable to detect copy move image forgery and splicing. The method was good to deal with multiple cloning. Though the method was found very accurate with a TPR (True Positive Rate) of 100\%, but failed to detect forgery in flat regions [11]. Baina Su and Zhu Kaizhen (2012) gave a new method based on LPP (Locality Preserving Projection) and SIFT features. The computation time was reduced due to dimension reduction property of LPP while still maintaining the advantages of SIFT i.e., robustness to rotation, scaling and JPEG compression [12]. Jaberi et al., (2013) proposed a method based on more powerful key-point based features Mirror Invariant Feature Transform (MIFT) to detect and locate the duplicated region in an image. MIFT features share all the properties of SIFT features and also exhibit robustness against mirror reflection transformations. The proposed method resulted in reducing the False Positive Rate (FPR) and False Negative Rate (FNR) of the detection results [13]. Kunlun Li et al., (2013) proposed an algorithm for detecting copy-move forgery using the idea of PCA-SIFT along with k-nearest neighbor. The proposed method possessed more detection accuracy and reduced time complexity than SIFT. It was also successful in handling with geometric transformations in the copied region. However, the proposed method needed some improvements to define the tampered region more precisely [14]. Although SIFT is robust against geometric transformations and is highly accurate in detecting tampered region in non-flat region. However, it is not able to detect flat copied region. So, Mohamadian Z. et al. (2013) proposed a method using SIFT features and Zernike moments to detect flat as well as non-flat regions copied areas. SIFT detection method was used on the complete image and resulted in forgery detection of non-flat regions whereas Zernike detection method was used in regions where SIFT was incapable to find key-points, thus detecting forgery in the complete image [15]. Hashmi et al., (2013), combined DWT with SIFT to detect image forgery so as to take the advantage of dimension reduction property of DWT in addition to the advantages of SIFT like accuracy and robustness against geometric transformations such as scaling and rotation of the copied part. The proposed method took less time for computation and provided higher accuracy than SIFT [16]. Dyadic Wavelet Transform (DyWT) is shift invariant and captures the structural information in a better manner than DWT. Based on this, Anand et al., (2014) 
proposed a new method for detecting copy move image forgery by combining DyWT and SIFT. DyWT with SIFT was found more efficient as compared to DWT combined with SIFT in detecting copy move forgery. The proposed method was also robust against geometric transformations. Also, the proposed method resulted in higher True Positive Rate (TPR) than the previous methods [17]. Sudhakar.K et al., (2014) proposed a simple high-speed method to detect Copy Move Forgery based on SIFT and Chan-Vese's level set approach. This method reduced the time complexity of matching process and hence made the forgery detection process faster [18]. Takwa Chihaoui et al. (2014) proposed a hybrid approach based on SIFT and SVD to detect copy move forgery in digital images. SIFT was used to detect and extract the key-points from the image and Singular Value Decomposition (SVD) was used as matching algorithm. The proposed method was found robust to geometrical transformations and resulted in better performance in detecting duplicated regions with less false matching [19]. Zhang et al., (2014) proposed a new method to detect image copy-move tampering using SIFT and bi- coherence features. In this paper, SIFT was used to detect and extract key-points. 3PCC (three Point Centre Clustering) method was used to make clusters of the key-points belonging to original region and the tampered region. RANSAC (Random Sample Consensus) algorithm was adopted to determine the geometrical transform between the source and duplicated regions. Bi-coherence features were extracted from the two matched regions to register the original region and the tampered region [20]. Liu et al., (2014) proposed an improved scale invariant feature transform (SIFT) based copy-move detection method, which combined Broad First Search Neighbors (BFSN) clustering and Color Filter Array (CFA) features. CFA features were used to find the inconsistencies at the edges of tampered regions which helped to discriminate between source zone and the duplicated ones in a particular image [21]. In the following figure 3.1, solid lined blocks

represents the compulsory fields whereas the dashed lined blocks are optional fields that depends on integrated method being used.

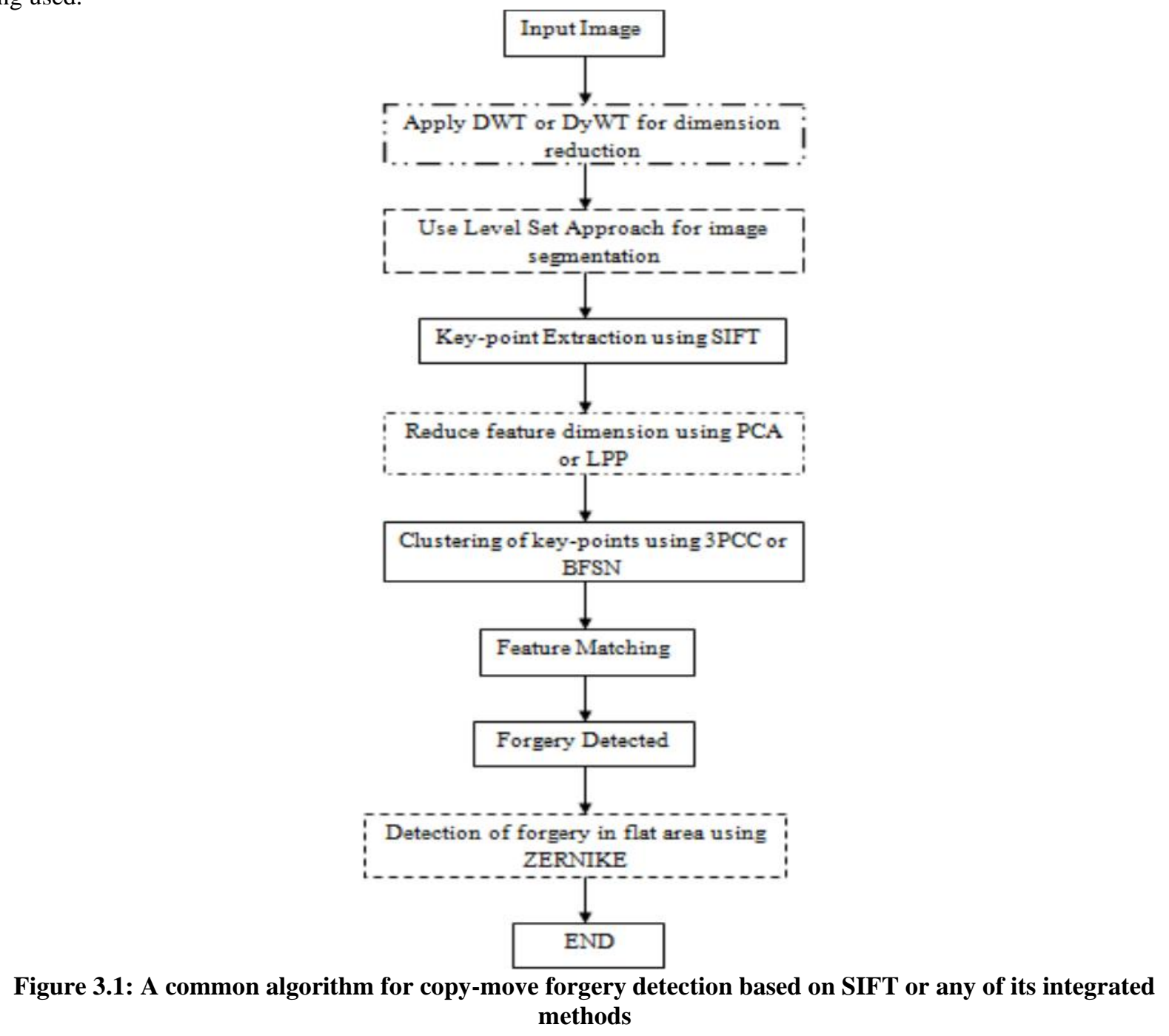

\subsection{SURF and its Integrated Methods}

SURF is another key-point descriptor which is widely used by researchers to detect copy move image forgery. SURF (Speeded Up Robust Features) is a robust local feature detector. It is an alternative to the SIFT descriptor. The standard version of SURF is several times faster than SIFT. Xu Bo et al., (2010) proposed a fast method to detect copy move forgery based on the SURF (Speed up Robust Features) descriptors. The proposed method was quick as well as reliable. The detection performance of this method was good even if the copied region experienced certain transformations such as scaling, rotation, noise, blurring and so on. However, further investigation was still needed to automatically locate the duplicate region and its boundary [22]. Shinfeng D. Lin and Tszan Wu (2011) proposed a fast and effective method to 
detect splicing and copy move image forgery. The proposed technique combined the discrete cosine transform (DCT) coefficients analysis by double JPEG compression with Speeded up Robust Features (SURF). The proposed method focused on JPEG format and performed well in localizing forgery. Moreover, it was able to detect multiple objects [23]. The key-point based methods (SIFT, SURF) are not able to detect forgery in flat regions as no key-point is extracted in such regions. To solve this problem, Zhang et al., (2012) proposed a technique combining the block matching based methods and key-point based methods. Authors proposed a SURF based method to detect forgery in non-flat regions and a block matching based method FMT (Fourier Mellin Transform) to detect forgery in flat regions [24]. Mishra et al., (2013) proposed a detection method based on speeded up robust features (SURF) and hierarchical agglomerative clustering (HAC). SURF detects the key-points and their corresponding features. Results of the proposed technique proved that both false positive rate (FPR) value and time required to detect forgery were reduced by using the proposed method. However, the proposed method had a drawback i.e. low true positive rate (TPR) value [25]. Hashmi et al. (2014) proposed two algorithms based on Speeded-Up Robust Feature (SURF) for copy-move forgery detection. In one algorithm it combined key-point based method SURF and block based method Discrete Wavelet Transform (DWT). In second it combined Speeded-Up Robust Feature transforms and Dyadic Wavelet Transform (DyWT). The results obtained from the proposed algorithms showed better precision rate as well as recall rate as compared to SURF. From the results obtained it was also concluded that the proposed algorithms are better than their counterparts both in terms of computational complexity and invariance to scaling and rotation, compression, noise addition and combination of these attacks. DyWT with SURF gave more robust method for copy move forgery detection than DWT with SURF due to shift invariance of DyWT [26]. In the following figure 3.2, solid lined blocks represents the compulsory fields whereas the dashed lined blocks are optional fields that depends on the integrated method being used.

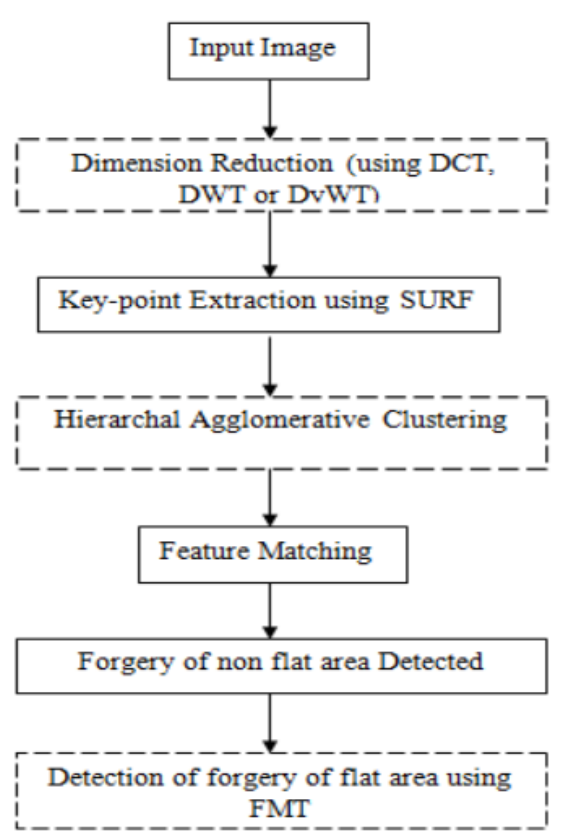

Figure 3.2: Algorithm for copy-move forgery detection based on SURF or any of its integrated methods.

\subsection{ORB and its Integrated Methods}

Jiming Zheng and Liping Chang (2014) proposed a new method based on ORB (Oriented FAST and Rotated BRIEF) which is based on the visual descriptor BRIEF (Binary Robust Independent Elementary Features) and the FAST (Features from Accelerated Segment Test) key-point detector. It is a fast binary feature descriptor and an alternative to SIFT and SURF. It used the binary descriptors of key points to detect region duplication forgery in images. Experimental results showed that the matching time of the proposed method was less than both SIFT and SURF. Accuracy of the proposed method for detecting region duplication is greater than SURF but less than SIFT. Also the proposed method required lower storage space. However, this method was not able to handle scaling of the copied region and also was not able to differentiate between source region and the tampered region [27]. To deal with scaling of the cloned region, Zhu $e t$ al. (2015), proposed a method based on scale space and ORB (Oriented FAST and rotated BRIEF). The proposed method not only detected duplicated regions but also determined the geometric transformations and post processing operations applied to the forged regions and removed the false matching. However, the method was still time-consuming for forgery detection of high-resolution images [28].

\section{DISCUSSION AND CONCLUSION}

The copy move image forgery detection is a promising frontier field of digital image forensics. Different block and keypoint based copy move image forgery detection methods have been developed by various researchers to cope up with image forgery problem. In this paper, the key- point based copy-move forgery detection (SIFT, SURF and ORB) and their integrated methods have been presented. It is observed that SIFT gives better result than SURF and ORB in terms of accuracy. Wheras ORB based detection methods take least time and hence are faster than above said methods. The performance of above said methods has been improved by researchers in terms of accuracy, computational efficiency and 
time taken for the detection of forgery by integrating them with key-point, block-based methods or any other appropriate algorithms. Future work may be focused to reduce FPR and detect copy-move image forgery in smaller regions by developing appropriate methods.

\section{REFERENCES}

[1].M. Sridevi, C. Mala and Siddhant Sanyam, “Comparative Study of Image Forgery and Copy- Move Techniques”, in Proc. of the Second International Conference on Computer Science Engineering and Applications (ICCSEA), vol. 166, pp. 715-723, May 2012.

[2].Kusam, Abrol Pawanesh and Devanand "Digital Tampering Detection Techniques: A Review", BVICAM's International Journal of Information Technology (BIJIT), vol. 1, no. 2, pp. 125-132, Jul. - Dec. 2009.

[3].Qureshi M. Ali and Deriche M., "A Review on Copy Move Image Forgery Detection Techniques", $11^{\text {th }}$ International Multi Conference on Systems, Signals \& Devices(SSD), pp. 1-5, Feb. $2014 .$.

[4].Kudke Swapnil H. and Gawande A. D., "Copy- Move Attack Forgery Detection by Using SIFT", International Journal of Innovative Technology and Exploring Engineering (IJITEE), vol. 2, no. 5, pp. 221-224, Apr 2013.

[5].Christlein Vincent, Riess Christian, Jordan Johannes, Riess Corinna, and Angelopoulou Elli, "An Evaluation of Popular Copy-Move Forgery Detection Approaches", IEEE Transactions on Information Forensics and Security, vol. 7, no. 6, pp. 1841 - 1854, Dec, 2012.

[6].Sekhar Resmi and A S Chithra, "Recent Block- based Methods of Copy-Move Forgery Detection in Digital Images", International Journal of Computer Applications, vol. 89, no. 8, pp- 28-33, Mar. 2014.

[7].Yu Liyang, Han Qi and Niu Xiamu, "Feature Point-based Copy-Move Forgery Detection: Covering the Non-Textured Areas", International Journal of Multimedia Tools and Applications, Springer, vol. 74, issue 4, pp. 1-18, 2015.

[8].Xunyu Pan and Siwei Lyu, "Region Duplication Detection Using Image Feature Matching", IEEE Transactions on Information Forensics and Security, vol. 5, no. 4, pp. 857-867, Dec. 2010.

[9].Huang Hailing, Guo Weiqiang and Zhang Yu, "Detection of Copy-Move Forgery in Digital Images Using SIFT Algorithm", Pacific-Asia Workshop on Computational Intelligence and Industrial Application (PACIIA), pp. 272 276, Dec. 2008

[10]. Ardizzone E., Bruno A. and Mazzola G., "Detecting Multiple Copies in Tampered Image", in Proceedings of IEEE 17th International Conference on Image Processing, pp. 2117-2120, Sep., 2010.

[11]. Amerini I., Ballan L., Caldelli R., Del Bimbo A. and Serra G., "A SIFT-Based Forensic Method for Copy-Move Attack Detection and Transformation Recovery", IEEE Transactions on Information Forensics and Security, vol. 6, no. 3, pp. 1099 - 1110, Mar. 2011.

[12]. Su Baina and Kaizhen Zhu, "Detection of Copy Forgery in Digital Images Based on LPP-SIFT", International Conference on Industrial Control and Electronics Engineering, pp. 1773 - 1776, Aug. 2012

[13]. Jaberi Maryam, Bebis George, Hussain Muhammad and Muhammad Ghulam, "Improving the Detection and Localization of Duplicated Regions in Copy-Move Image Forgery", 18th International Conference on Digital Signal Processing (DSP), pp. 1-6, Jul. 2013.

[14]. Li Kunlun, Li Hexin, Yang Bo, Meng Qi and Luo Shangzong, "Detection of Image Forgery Based on Improved PCA-SIFT", in Proceedings of Computer Engineering and Networking, vol. 277, pp 679-686, 2014.

[15]. Mohamadian. Z. and Pouvan A.A., "Detection of Duplication Forgery in Digital Images in Uniform and Nonuniform Regions", 15th International Conference on Computer Modeling and Simulation (UKSim), pp. 455 - 460, Apr. 2013.

[16]. Hashmi Mohammad Farukh, Hambarde Aaditya R. and Keskar Avinash G., "Copy Move Forgery Detection using DWT and SIFT Features", $13^{\text {th }}$ international Conference on Intelligent Systems Design and Applications (ISDA), pp. 188 - 193, Dec. 2013.

[17]. Anand Vijay, Hashmi Mohammad Farukh and Keskar Avinash G., “A Copy Move Forgery Detection to Overcome Sustained Attacks Using Dyadic Wavelet Transform and SIFT Methods", in Proceedings of Intelligent Information and Database Systems, Springer, vol. 8397, pp. 530- 542, 2014.

[18]. Sudhakar K., Sandeep V.M. and Kulkarni S., "Speeding-up SIFT based Copy Move Forgery Detection Using Level Set Approach", International Conference on Advances in Electronics, Computers and Communications (ICAECC), pp. 1-6, Oct. 2014.

[19]. Chihaoui Takwa, Bourouis Sami and Hamrouni Kamel, "Copy-Move Image Forgery Detection Based on SIFT Descriptors and SVD-Matching”, First International Conference on Advanced Technologies for Signal and Image Processing (ATSIP), pp. 125-129, Mar. 2014.

[20]. Zhang Ju, Ruan Qiugi and Jin Yi, “Combined SIFT and Bi-Coherence Features to Detect Image Forgery", 12th International Conference on Signal Processing (ICSP), pp.1859 - 1863, Oct. 2014.

[21]. Liu Lu, Ni Rongrong, Zhao Yao and Li Siran, "Improved SIFT-based Copy-move Detection Using BFSN Clustering and CFA Features", Tenth International Conference on Intelligent Information Hiding and Multimedia Signal Processing, pp. 626 - 629, Aug. 2014.

[22]. Bo Xu, Junwen Wang, Guangjie Liu and Yuewei Dai, "Image Copy-move Forgery Detection Based on SURF", International Conference on Multimedia Information Networking and Security (MINES), pp. 889 - 892, Nov. 2010.

[23]. Lin Shinfeng D. and Wu Tszan, “An Integrated Technique for Splicing and Copy-move Forgery Image Detection”, 4th International Conference on Image and Signal Processing (CISP), vol. 2, pp. 1086 - 1090, Oct. 2011.

[24]. Zhang Guang-qun and Wang Hang-jun, "SURF- based Detection of Copy-Move Forgery in Flat Region", International Journal of Advancements in Computing Technology (IJACT), vol. 4, no. 17, Sep., 2012. 
[25]. Mishra Parul, Mishra Nishchol, Sharma Sanjeev and Patel Ravindra, "Region Duplication Forgery Detection Technique Based on SURF and HAC", The Scientific World Journal, 8 pages,

[26]. Hashmi M.F., Anand V., Keskar A.G., "A Copy- move Image Forgery Detection Based on Speeded up Robust Feature Transform and Wavelet Transforms", International Conference on Computer and Communication Technology (ICCCT), pp. 147 - 152, Sep. 2014

[27]. Jiming Zheng and Liping Chang, "Detection of Region-duplication Forgery in Image Based on Key Points' Binary Descriptors”, Journal of Information \& Computational Science, vol. 11, no. 11, pp. 3959-3966, Jul, 2014.

[28]. Zhu Ye, Shen Xuanjing and Chen Haipeng, “Copy-Move Forgery Detection Based on Scaled ORB ”, Proceedings of Multimedia Tools and Applications, vol. 75, no. 9, pp. 1-13, Jan. 2015. 